\title{
WILD HOPPER Prototype for Forest Firefighting
}

\author{
https://doi.org/10.3991/ijoe.v17i09.25205 \\ Ahmed Refaat Ragab ${ }^{1(\varpi)}$, Mohammad Sadeq Ale Isaac ${ }^{2}$, Marco A. Luna ${ }^{2}$, \\ Pablo Flores Peña ${ }^{3}$ \\ ${ }^{1}$ University of Carlos III, Madrid, Spain \\ ${ }^{2}$ Polytechnic University of Madrid, Madrid, Spain \\ ${ }^{3}$ Drone Hopper Company, Madrid, Spain \\ a.refaatedrone-hopper.com
}

\begin{abstract}
In Europe, fire represents an important issue for a lot of researchers due to economic losses, environmental disasters, and human death. In the last decade, the European parliament sheds light upon this problem by dealing with the community project" Forest Focus". Thus, researchers and scientific research departments of European companies begin to work on solving and creating different techniques to deal with such a problem, these research centers found that the most attractive and accurate way of solving such a problem was using an Unmanned Aerial Vehicle (UAV). In this paper, the research center at Drone Hopper Company analysis the deficiencies for forest fire fighting systems, in order to start designing its new prototype of a special drone named WILD HOPPER, solving all the shortcomings of similar systems. This paper is the first of a group of research papers that will take place during designing and producing our WILD-HOPPER system.
\end{abstract}

Keywords—Forest Fire, European parliament, UAV, WILD HOPPER

\section{Introduction}

Forests in Europe cover around 1000 million hectares of its area, which can be thought of as 20 times the size of Spain, 12 times the size of Greece and 34 times the size of Germany, where Spain comes as the fifth European country with total forest area $36.7 \%$ of its total area, also the proportion of forests in relation to Europe's total land area is $47 \%$, forests play an important role in moderating climate change by absorbing carbon from the atmosphere, also its importance is clear at the regulation of water, air quality, and the provisioning of a plethora of wood products [1]. Fires are the predominant threat to forests than any other natural disaster like rapid winds, parasites, and frost [2]. The huge responsibility of these fires depends on humans (over 95\%), as stated in the European Fire Information System (EFFIS) report [3]. Thus, Fire became the main threat to European forests, and as a result, a complete fire-exclusion policy was developed based on very strong, sometimes military-like, fire-suppression organizations. Also, the "Council Regulation (EEC) No. 2158/92 on the protection of the Community's forests against fire" (and the regulations No. 1170/93, No. 804/94, No. $1727 / 1999$, No. 805/2002), focused on prevention significantly improving knowledge 
about forest fires, and to improve forest protection by reducing fire flow and burned surfaces. This regulation was substituted by "Regulation (EC) No. 2152/2003 and the complementary regulations No. 2121/2004 and No. 1737/2006, for the Council concerning monitoring of forests and environmental interactions in the Community (Forest Focus) and the European Parliament. "Forest Focus" was a Community project, running from 2003 to 2007, mainly focused on protecting forests against atmospheric fires and pollution. Although these problems are quite common throughout the world, they are all common with the danger of fire, this main problem can be seen as a hazard that affects most of the European countries, which may cause and impact changes in critical climatic and weather conditions. A lot of researchers' work was in the field of monitoring and detecting Wildfires in a European forest, by using several techniques such as thermal sensors, satellite data, and Unmanned Aerial vehicles (UAVs). Joaquim de Sousa et al. (2020) [4], designed UAV onboard footage with Fire Detection and Monitoring algorithm, but they face a shortage in their system due to the limited processing power of the onboard computer, this limitation of power affects the overall system with lower video resolution. Also, Amit Sharma et al. (2019) [5], designed a new system using UAV and wireless sensor network distributed among specific area to gather data for temperature, humidity, light, and smoke and transfer it to the UAV, the system shortage was the communication process, where they face a problem with the UAV network to track the ground-based network the (WSN) for the restriction of a line of sight. In addition, Omar A. Saraereh et al. (2020) [6], added a new network system called UAV-enabled LoRa network, the major aspect of their system was to extend the network coverage offered by the base station to a set of mobile ground nodes using relaying gateways installed on the UAVs, but their work was restricted due to the power constraints, also no thermal camera or sensors were added to their system. Moreover, Sezgin Kilic et al.(2019) [7], proposed a conceptual model for mitigating forest fire risk using autonomous and self-adaptive unmanned aerial vehicles, their main research point was solving the routing problem of UAVs for detection forest fires, their work was to design a dispatching rule in a self-adaptive routing of UAVs for detecting forest fires, by calculating fire probabilities, their work has a lack of combining the new powerful technologies and their results were more theoretical. From the previous, it is cleared that a lot of work and researches were developed in the field of UAVs and firefighting and detecting, through the huge benefits of UAVs, but there wasn't an accurate solution or a new independent system to solve such a problem. And as an action from the research center at Drone Hopper Company and Universidad Carlos III de Madrid (UC3M), Spain. We are going to shed light upon a critical circumstance in order to design a new powerful independent system for forest fire fighting, by pointing through the system requirements, safety requirements, and operational requirements. This paper is organized as follows, Section two highlights the limitation of the existing firefighting technologies; Section three shows the plan for Drone Hopper through implementing the roadmap of the WILD HOPPER, clarifying the importance of the safety requirements, and showing the software development for WILD HOPPER; Section four discuses the dynamic model for WILD HOPPER, and how to deal with the payload of the system during the mission; Section five demonstrates the results with corresponding discussion; and finally Section six concludes the whole paper. 


\section{Limitations of Current Firefighting Technologies}

Alternatives to fighting wildfires, as illustrated in "Fig. 1", include manned aerial firefighting like fixed-wing aircraft, helicopters, smoke jumpers and rappelers, and ground firefighters. Unfortunately, all of these options put human lives at risk. Current aerial means cannot work at night, limiting the operation time to an average of 12 hours. Also, their maneuverability is restricted and the way they release water is highly inefficient since they simply drop the water by gravity at a certain speed. Besides, water release is hampered by the distance at which they can approach: fixed-wing can't reduce their horizontal speed below the stall speed, which is typically above $100 \mathrm{Km} / \mathrm{h}$, necessary to get close and helicopters cannot get closer than $50 \mathrm{~m}$ since they might fan the fire with their rotor wake. Moreover, they need a landing point, which implies several operational issues like the obligation of returning to the landing point to be water and/or fuel recharged for another mission. Besides all their technical limitations, their capital and operational costs are extremely high. Although, through our research, we found that almost all the previous research work on this field full into one of main three categories respectively, theoretical way of research as in [8], practical way put with limited resources by using single lightweight UAV, focusing their work on sensing and thermal cameras as in [9], and finally using a swarm of multi mini-drones as in [10].

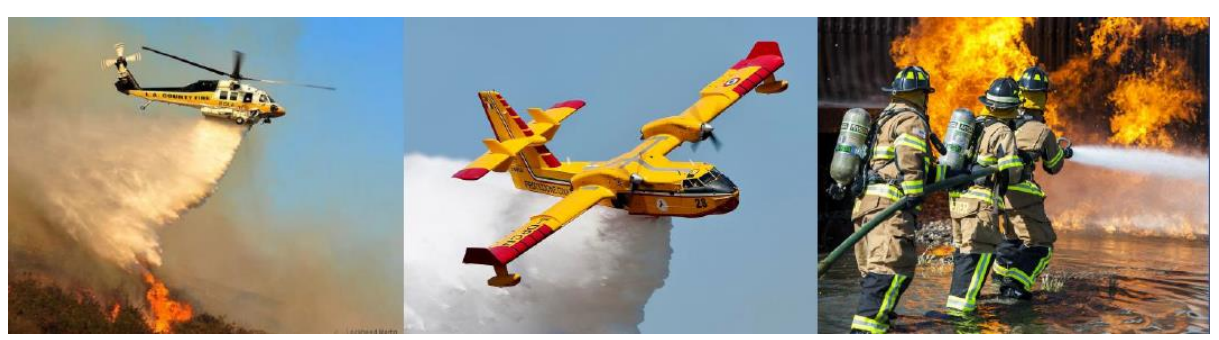

Fig. 1. Ordinary fighting wildfires techniques.

In [9] the researchers stated that the main requirements for such a system can be considered in all-weather suitability, in the mean of day and night functionality, selflocalization using a common terrain reference model for automatic geo-localization, navigational autonomy by using GPS and IMU, cooperation behavior with other UAVs, gathering the payload to be only for sensors (fire perception purposes), and finally the availability by equipping the communication devices onboard. Although, through our research, we found that almost all the previous research work on this field full into one of main three categories respectively, theoretical way of research as in [8], practical way put with limited resources by using a single lightweight UAV, focusing their work on sensing and thermal cameras as in [9], and finally using a swarm of multi minidrones as in [10]. In [9] the researchers stated that the main requirements for such a system can be considered in all-weather suitability, in the mean of day and night functionality, self-localization using a common terrain reference model for automatic geolocalization, navigational autonomy by using GPS and IMU, cooperation behavior with other UAVs, gathering the payload to be only for sensors (fire perception purposes), 
and finally the availability by equipping the communication devices onboard. Also, they stated that their platform is fixed wing micro - UAV, with an electric system, and the total duration time is 3 hours, their final conclusion was that their system can help the decision-maker to calculate only the fire parameters, but not fighting the fire itself. In addition, Elena Ausonio et. al [10] stated that their system depends on the use of a swarm of hundreds of UAVs to simulate the rain effect as shown in "Fig. 2".

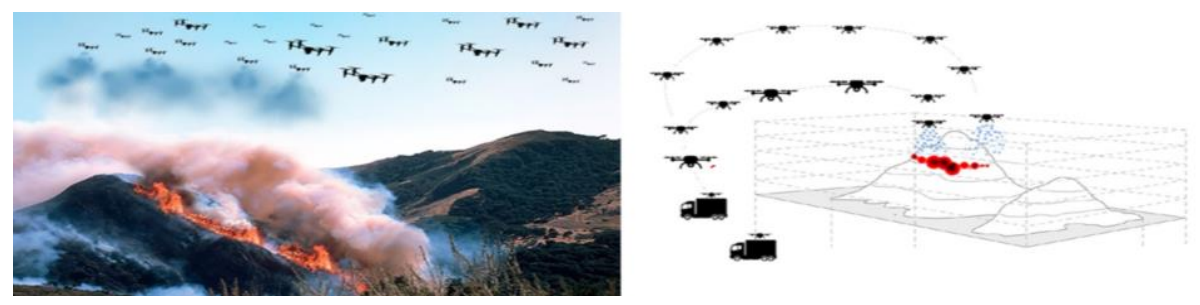

Fig. 2. Representations of firefighting system based on the use of a swarm of collaborative UAVs [10].

However, the use of UAVs for wildland firefighting operations (water release to extinguish the fire) as an alternative and/or complement to manned aerial vehicles, is not yet operative. The opportunity for firefighting drones is enormous, but there are still technological limitations preventing their use, as they must involve, which allows using an unmanned platform capable to transport heavy payloads and an extremely precise and effective water release system to be released over the fire. We have already overcome these technological barriers by developing our patented control system and the water mist jet creation mechanism.

\section{Roadmap for WILD-HOPPER}

Drone Hopper's roadmap for the new powerful forest fire fighting system named WILD-HOPPER, this system depends upon fundamental requirements to be followed by the designing and development process, in this paper we are going only to highlight some fundamentals during our research to have our proto-type. WILD-HOPPER roadmap depends upon three main requirements, respectively system requirements, which includes those requirements that must be present on the system necessary for its use such as hardware components and software resources, operational requirements, which includes those requirements focused on how the system will be operated by users including conditions under whom the system will be running, and finally Safety Requirements, which includes those requirements that must be followed to reduce any given risk.

\subsection{System Requirements}

The maximum takeoff weight (MTOW) of WILD-HOPPER will be $\leq 1200 \mathrm{~K} \mathrm{~g}$, this MTOW must be achieved using maximum continuous power (normally around $75 \%$ of 
maximum power in combustion engines) and may be lower if other requirements impose it. Also, the Maximum payload (MPL) of WILD-HOPPER will be $\geq 600 \mathrm{~K} g$ with a Flight Time (FT) $\geq 1$ hour, $\geq 300 \mathrm{Kg}$ with a FT $\geq 2$ hours, $\geq 100 \mathrm{~K}$ g with a FT $\geq 3.5$ hours, or $\geq 50 \mathrm{~kg}$ with a FT $\geq 5$ hours, where the MPL is an end goal, and for initial releases, lower values of MPL and FT are acceptable, this requirement will make WILD-HOPPER a high-capacity drone. In addition, the internal combustion engines will be used as the main power plant, whereas the number of engines should be the minimum possible due to aircraft attitude control requirements, to be able to overcome the limitations of conventional electrical systems in autonomy and payload. Besides that, the lift of the aircraft will be guaranteed by 6 rotors, where the rotors will be arranged in 2 parallel rows to increase performance and eliminate risks, as shown in "Fig. 3".

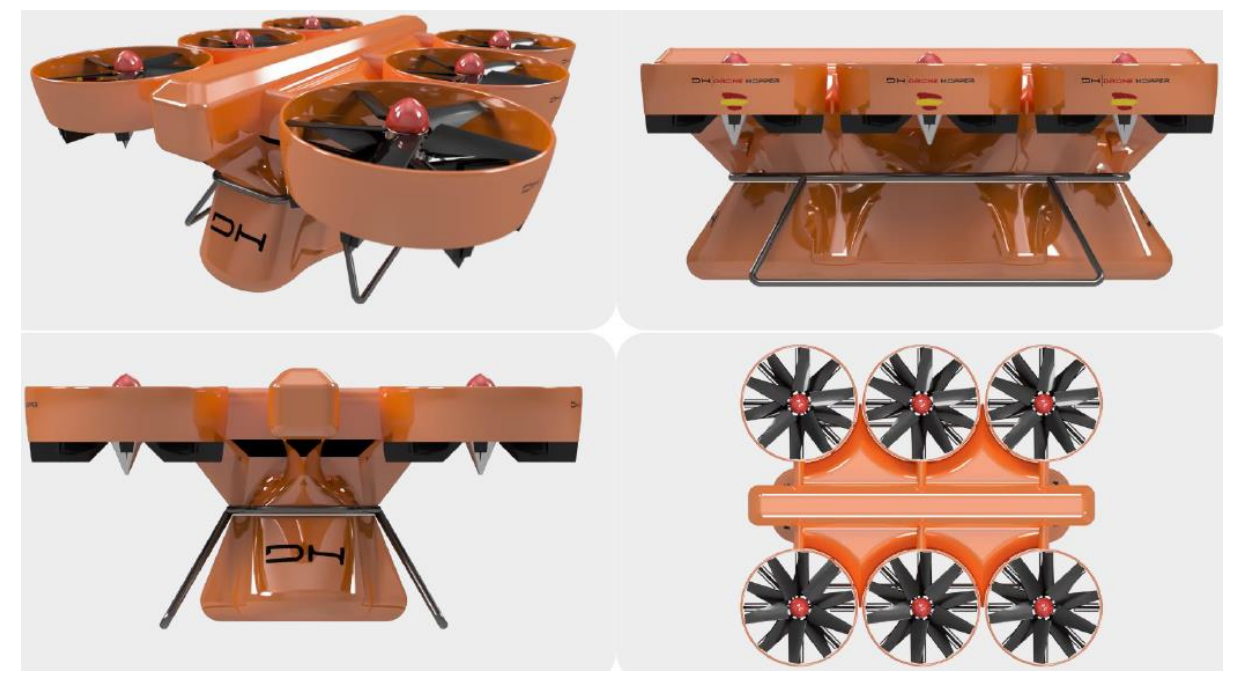

Fig. 3. WILD-HOPPER prototype.

And for more information about our WILD-HOPPER you can visit WILDHOPPER. Through "Fig. 4 and 5", the steps of designing and developing the prototype of the WILD-HOPPER and building our $600 \mathrm{~K} g$ heavy duty WILD-HOPPER platform. 


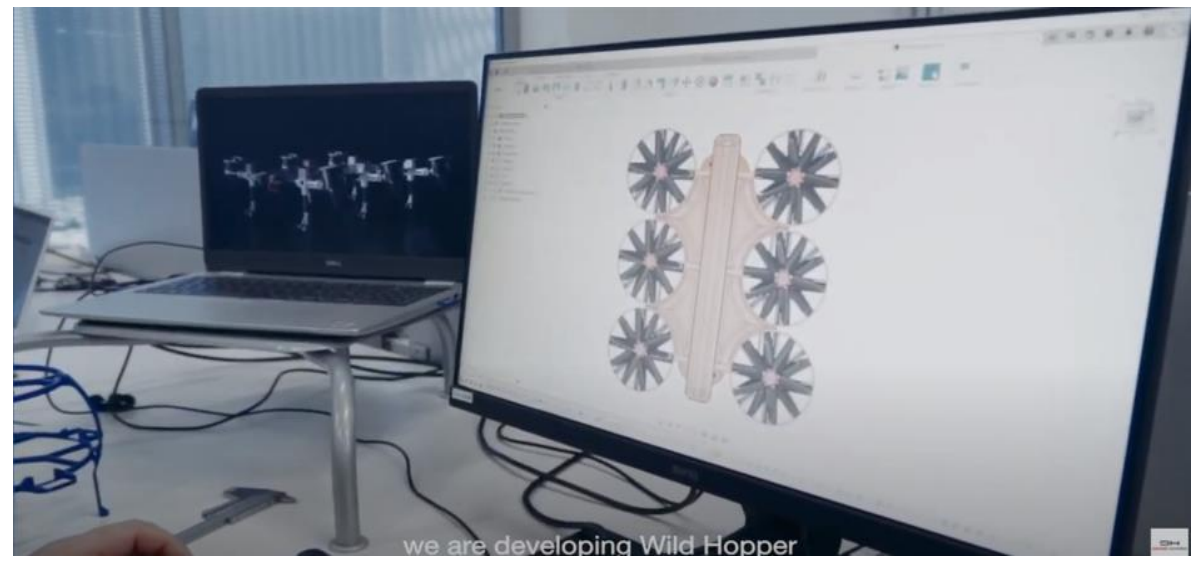

Fig. 4. Designing WILD-HOPPER prototype.

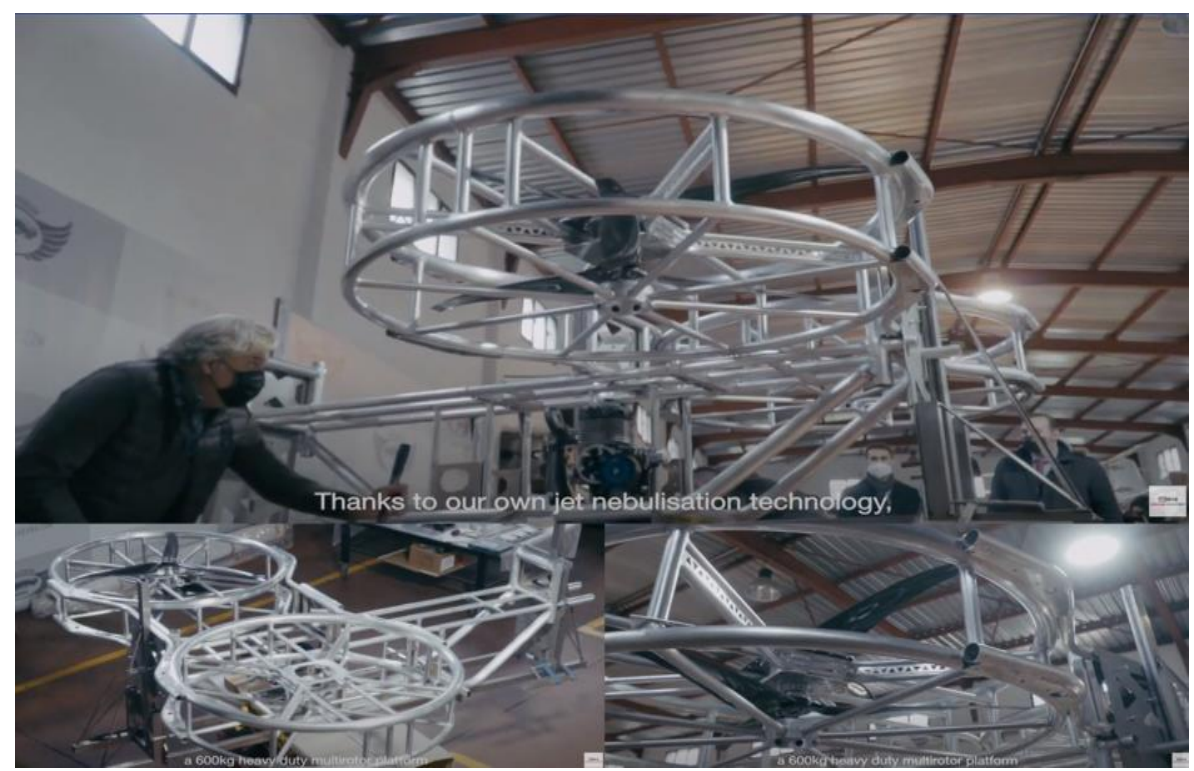

Fig. 5. Building WILD-HOPPER.

\subsection{Operational Requirements}

WILD-HOPPER will be able to operate at a fixed point and move through the air in a controlled manner as shown in "Fig. 6". 


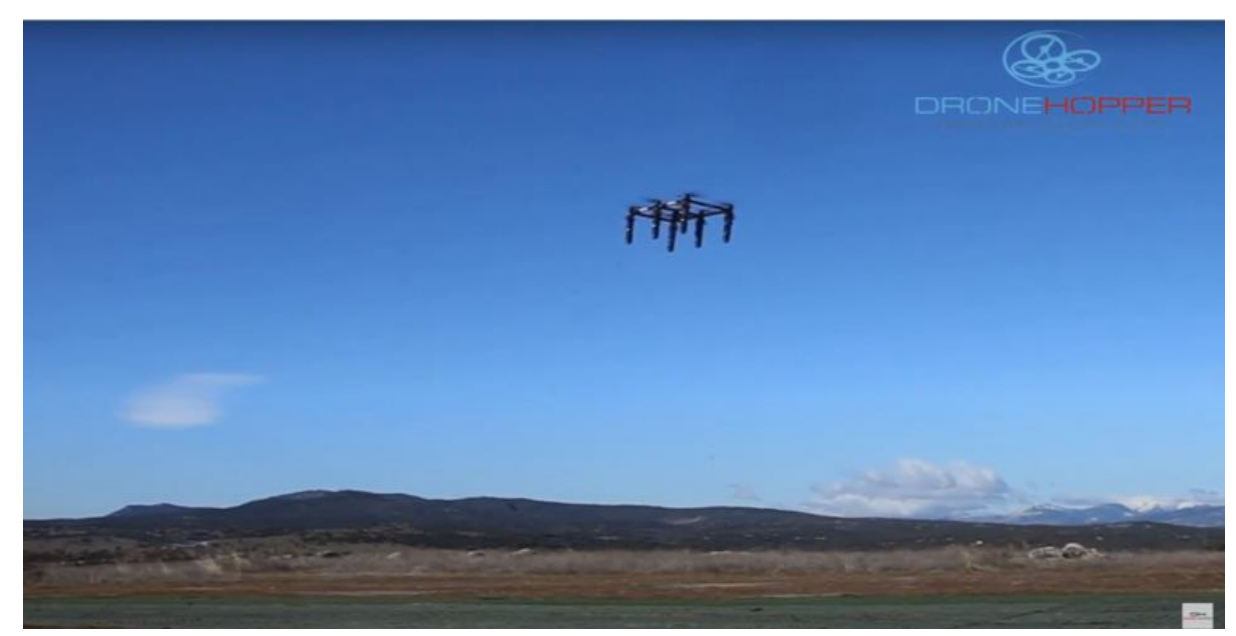

Fig. 6. WILD-HOPPER prototype operational test.

The Combustion engines of the WILD-HOPPER have powerful characteristic, which can be considered in the electronic start, generator, cooling system, monitoring system, injection, and engine control unit (ECU), and through our prototype design, we have made several calibrations. The objective of these calibrations is to document and record all activities, adjustments, and incidents detected from the procedures carried out in the prototype of WILD-HOPPER by the research center of Drone Hopper, to carry out a flight configuration and an adjustment of the control loops that allow a stability flight for WILD-HOPPER prototype. During this process, all the information related to avionics, control, and system calibration is recorded and documented. The system adjustment and calibration process were carried out in different phases, guaranteeing safety in all operations and tests. These adjustment phases are gathered in a Virtual Reality Simulation (VRS) file which is the principle setting for every real flight test. This file includes controller loops, geofence setting, servo calibration, governor calibration, rotor settings, Kalman Filter gains, several Primary Flight Displays (PFDs), video recorder settings, and so forth. Figure 7, shows the RPMs for rotor 4-adjusting governor with load, where the governor adjustments have been made on the bench by installing WILD-HOPPER prototype in plus configuration, leaving arms 2 and 4 free of movement to make the governor adjustments with weight load on the rotors. The governor control loops have been adjusted using $6 \mathrm{~K}$ g of weight as ballast in each arm $( \pm 5 \mathrm{~K} \mathrm{~g}$ of weight per rotor) to adjust that the autopilot maintains constant RPMs with oscillations less than \pm 100 RPM s. 


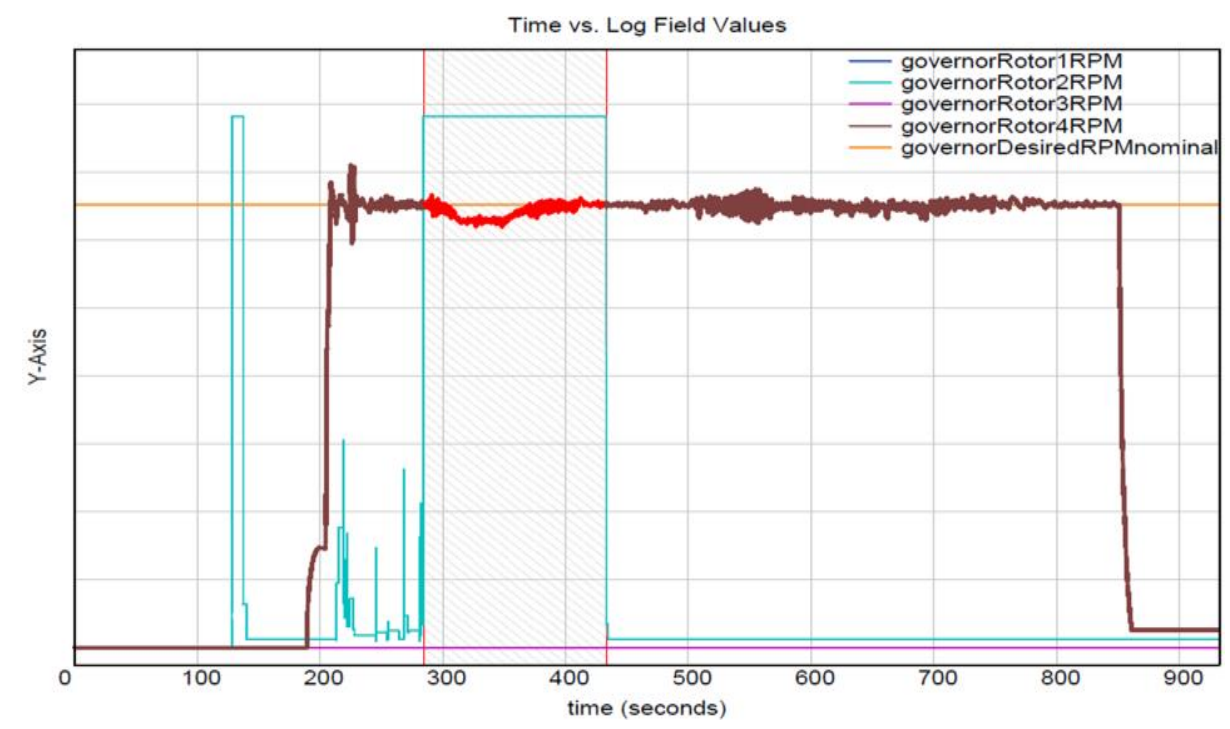

Fig. 7. Rotor-4 RPM, Adjusting governor with load.

The objective of these calibrations is the documentation and recording of all the actions, adjustments, and incidents detected from all the procedures carried out in the prototype of WILD-HOPPER by the research center of Drone Hopper, to carrying out a flight configuration and an adjustment of the control loops that allow a stability flight for WILD HOPPER prototype. Also, WILD HOPPER is designed to be easily controlled in both manual and an automatic mode, where the pilot can take control in manual mode throughout the entire operating range, besides that the latency in manual mode is less than $100 \mathrm{~ms}$ to ensure the control, as shown in figure 8. WILD HOPPER is easily transportable in a standard compartment $(2.27 \mathrm{~cm}$ high $\times 2.45 \mathrm{~cm}$ width $)$. Also, it incorporates provisions to integrate a standard cargo bay that allows the integration of different payloads with different anchorage systems, with dimensions $3 \mathrm{~m} \times 2 \mathrm{~m} \times 1$ $\mathrm{m}$. Besides that, WILD-HOPPER incorporates an exterior lighting system that complies with the applicable regulations for UAVs of these characteristics. WILD HOPPER incorporates aerodynamic (and ideally structural) around the propellers to increase thrust and protect operators. Through designing our prototype of WILD HOPPER, we take into account the possibility of evolving to alternative power plants without major structural changes (electric, hybrid, and hydrogen). 


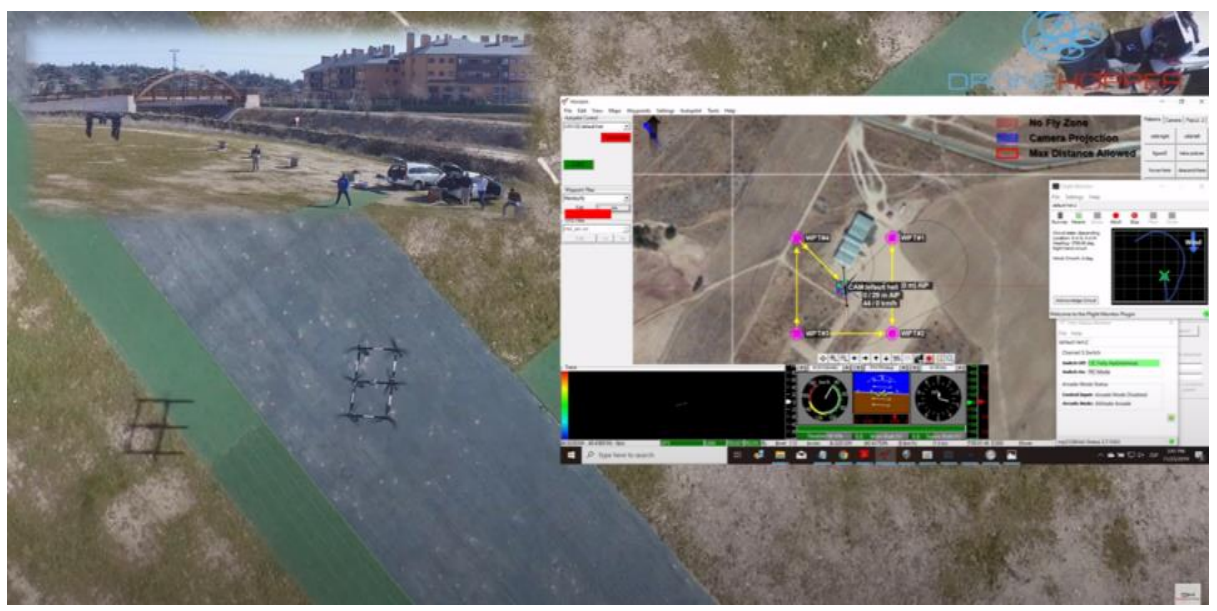

Fig. 8. Controlling WILD-HOPPER prototype testing in both manual and autonomous modes.

\subsection{Safety Requirements}

Through designing our prototype for WILD-HOPPER, the safety requirements where our aim in order to meet accurate safety issued by Regulation (EU) 2018/1139)[11], starting by dealing with the attitude control of WILD-HOPPER which is designed to be through two dissimilar and independent systems, where the System can operate in parallel and/or sequentially way. Also, it was taken into account the failsafe mode for WILD-HOPPER, so that WILD-HOPPER can land safely in the event of an engine failure or any critical situation during its missions, such as in case of losing communications or malfunction of the autopilot (Back home / Kill Switch by-passing the autopilot). Besides, WILD HOPPER is designed to be the safest system that deals in such a mission, by designing its communication system to have two independent and dissimilar control links, gathering both the ground control station and the autopilot. In designing our prototype for WILD-HOPPER we insist to make the system safer and secured by making both the autopilot and the GCS have a geofence activated on all flights, and for this reason, if the geofences are not activated the system will not allow flight. And for the purpose of working at different environment field such as working over electric or magnetic fields, and over different terrain that can affect any other systems by the mean of interference or jamming, WILD HOPPER is designed to incorporate protections against attempted unlawful interference of communications (Spoofing and Jamming). The most important issue in designing WILD HOPPER is that engines and fuel tanks are confined in non-fire permeable compartments, also we are planning to add an extinguishing self-system to make it more secure. 


\subsection{Software Development}

Since WILD HOPPER is a platform focused on critical task performance as firefighting, it is necessary to develop efficient and user-friendly software for flight operations. Nowadays, there is a lot of options for GCS software that offer multiple options for mission planning, control, monitoring, flight patterns performance, and emergency response as: Mission Planner[23], QGround Control[24], UgCS[25], Horizon[26], and others. To meet the operational and safety requirements of the project, it has been developed complementary GCS software using wxWidgets $\mathrm{C}++$ libraries (open source) [27] and autopilot SDK. This software will perform three main tasks:

- Safe parameters configuration

- Redundant alarm system

- Fast response flight pattern execution

For this, different software applications will deal with each task, this will be cleared through the next sections.

Safe parameters configuration. This application helps the operator to configure a safe point location, that is, a point with fixed coordinates and fixed altitude to go in case of something unexpected happens. Additionally, the user has the option to configure the bingo time setting (after this time, the aircraft will return to the initial point and land). The user interface is shown in "Fig. 9".

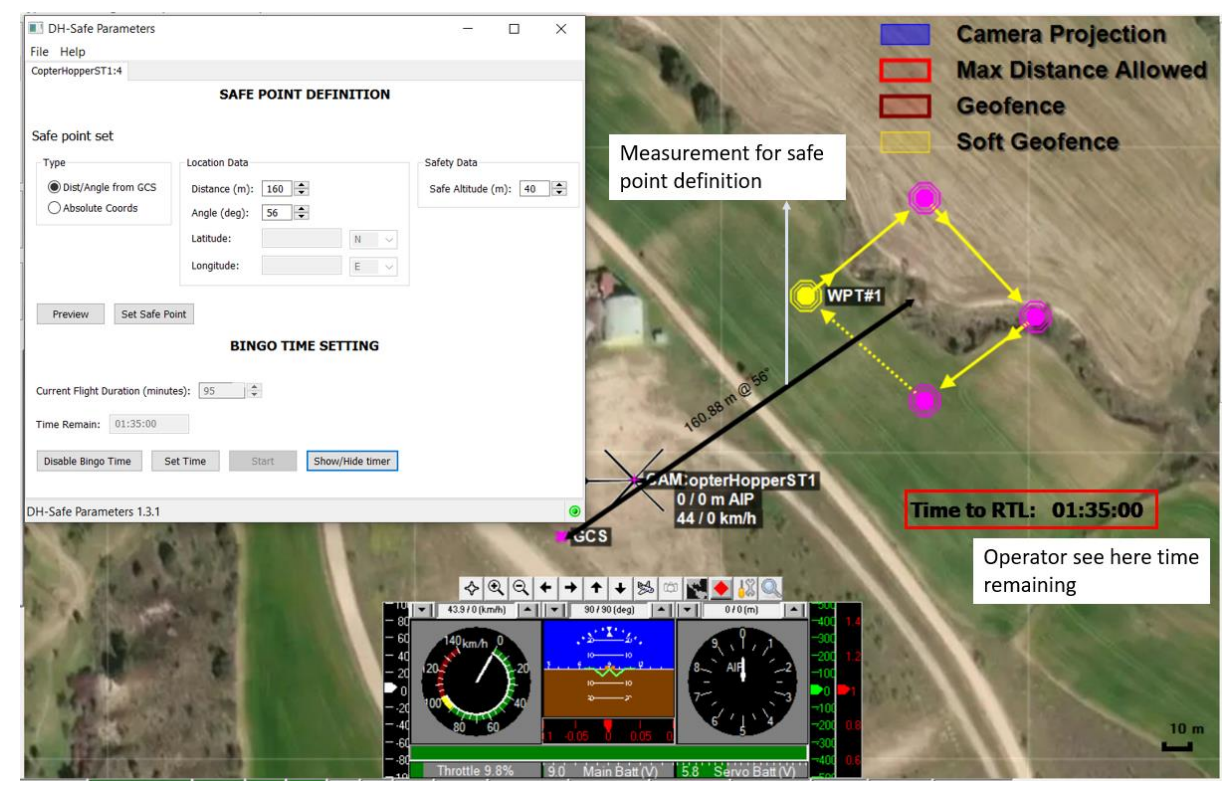

Fig. 9. Software interface for safe parameters Configuration.

For safe point definition, the software reads the GCS coordinate location as part of autopilot parameters, then it takes as input the desired location (distance and angle from 
GCS), calculates the coordinates of the location of the safe point, and save them into the autopilot. The simulated flight performance is showed in "Fig. 10".

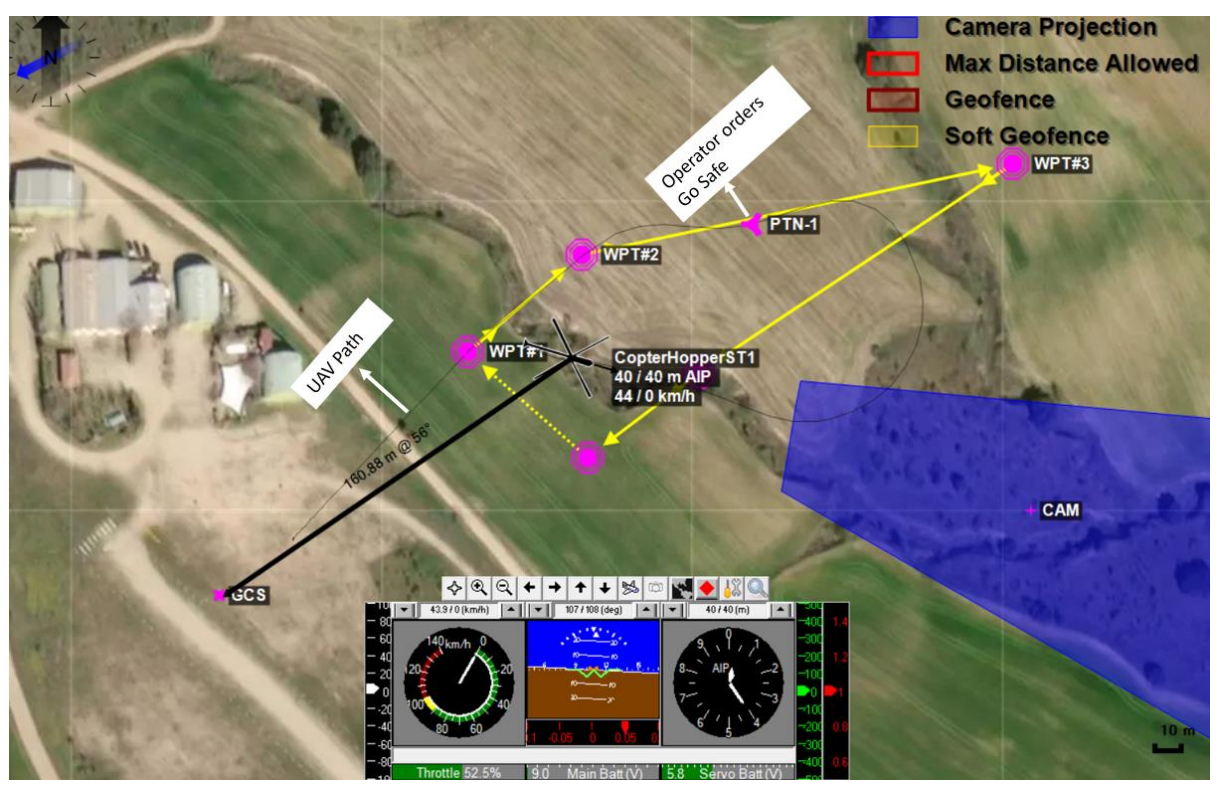

Fig. 10. Performance of the safe parameter application.

Additionally, for the bingo-time function, the application takes as input the desired flight time and saves it into the autopilot. When the aircraft is in flight, the autopilot reads its clock and performs flight time calculations. Finally, when the flight time defined by the user has expired, it orders to the system Return to Launch Point (RTL) and Land.

Redundant alarm system This software consists of a customized alarm system. In this system, the user can quickly determine the problem with the UAV when it is detected. If errors occur during working, the software groups them into user-friendly error categories as GPS, IMU, RC, Mission, Flight limits, and others. Depending on the alarm risk level (warning or error), it is showed in red or yellow color on its corresponding category. If the operator wants to check more details of the issue, a new screen appears, and then when the alarm cell is clicked; it shows the details of the error and the related sensors. For better understanding, "Fig. 11" shows an example. Considering that each GCS software has different ways to show the errors during the operation, the approach presented here provides additional and customized information focused on the operator requirements. 


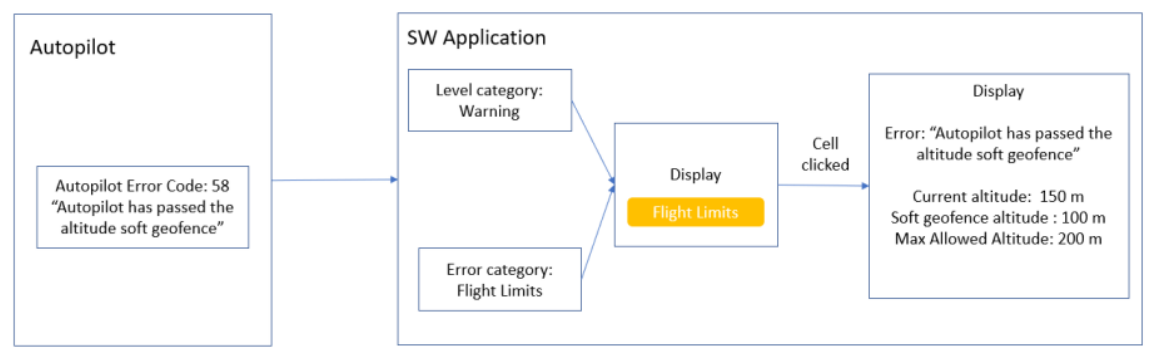

Fig. 11. process of error monitoring interface.

Also, the autopilot was programmed to perform different control actions when an error is present, whereby, this tool is not a distraction for the user.

Fast response flight pattern execution. For emergency response, we developed a new application for in-flight fast flight pattern planning. These flight patterns can be executed at any time during the main mission execution and return to the main mission easily. Also, it can be customized by the GCS operator. Focused on aircraft monitoring and firefighting tasks, this application enables to the user execute the following flight patterns:

- Orbit: Circle flight with a tangential heading.

- Funnel: Circle flight with a centripetal heading.

- Racetrack: Four waypoints flight.

- Search: Spiral flight.

- Grid: Flight into streets.

For circle and funnel patterns, the radius and the direction of rotation can be configured, while in racetrack pattern, the operator can configure the dimensions and the angle of the racetrack, and the turning sense. The Search pattern enables the user to customize the radio of the spiral and the width of the streets. Finally, in the Grid pattern, the dimensions, angles, and the width of the streets can be customized. "Fig. 12" shows a flight path of an application test.

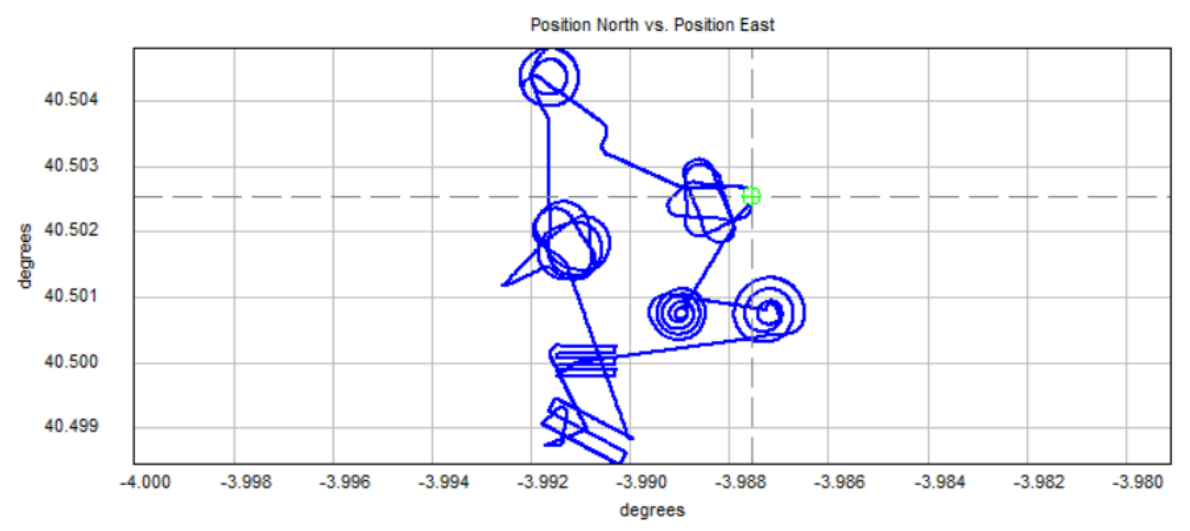

Fig. 12. Flight path generated using the software application. 
The results of the performance evaluation for this application are presented in the Results section. Additionally, more details of implementation and video results can be found at URL: https://drone-hopper.com/dh-mission-wizard/.

\section{Dynamic Model of the WILD HOPPER}

The dynamic model of the WILD-HOPPER has been changed many times to be customized upon the main objective. Meanwhile, we were keen into finding solutions with more novelty and several layers of control as redundancy. At first blush, the WILD-HOPPER was supposed to be an hexa-copter without flap, but with Electrical Ducted Fans (EDFs); this designation, however, fails in harsh situations, when the fire is blaming catastrophically, several maneuvers will be needed and if an engine burns, this would be hard to do controlled landing. Therefore, in second, we have made an architecture to be totally immune for one engine fails, flaps are provided to compensate the stability. Then finally, to augment more thrust force, redundant side EDFs are utilized, this manner even is promising for yaw rotation, due to the rotating joint of EDFs. Considering such a heavy drone, the fuselage made with a semi-rectangular geometry and a quite aerodynamic customization which are step by step simulated in ANSYSFLUENT software to be verified. In addition, propellers are designed to generate maximum feasible thrust force, this procedure is performed by Drone-Hopper research center, who are expert in charge of blade and turbine optimization to reduce the noise of rotation and minimize vorticity throughout the ducts which results in lesser unwanted yawing torque. To continue, about the ducts which are magnificent novelties of WILDHOPPER during the designation. This part is visible in "Fig. 13", brilliantly; they are modified to be short adequate for flap installment. According to the simulation results, flaps should be fixed out of the duct-cover to bring about sufficient lift. Supposing flaps were inside, the surface area would be smaller related to the ambient, and so result in lesser circulation stream which generates lift force. "Fig. 13 (a)" demonstrates the installment of four flaps (roll and pitch), presenting the main dimensions. Each flap is 14 $\mathrm{cm}$ in width and $51 \mathrm{~cm}$ in length; moreover as shown in "Fig. 13 (b)", it has equipped with dorsal to make the passing stream stick to the surface of the flap as much as feasible, which aims to reduce the drag force.

Furthermore, servos are used to rotate flaps are studied to maintain sufficient torque, here, one per flap; however, each pair of servos is disciplined to be commanded by the controller once. Consequently, WILD-HOPPER has 36 servos as many as flaps, but in action, three sets of flaps are performing moments, i.e., every six ones generate a unique moment, including rolling, pitching, and yawing, therefore, the controller structure is configurable easily. 

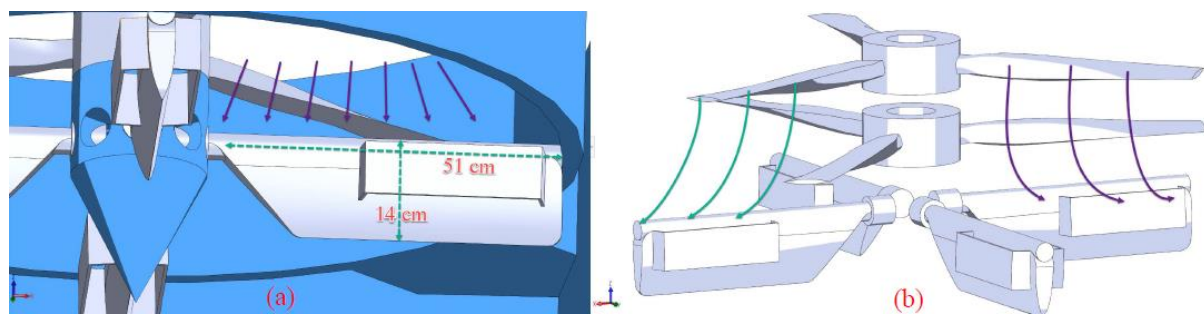

Fig. 13. Flaps arrangement below the coaxial propellers, outside of the duct to influence maximum.

Finally, side EDFs are manufactured by Schuebeler which are used augmenting both thrust and yawing moment. The configuration of EDFs is still under study to be optimized for saving energy. Hereafter, all generalized mathematical formulas are brought to clarify the dynamic system of WILD-HOPPER. "Fig. 14" displays the direction of rotation of each propeller as three positive (yellow ones) and three negative (green ones), which are reversed to the direction of coaxial pairs below. Moreover, the three main frameworks are brought; considering Body frame concordant with the Inertial coordinate system and contradicted to the North-East-Down (NED) frame, transformations are drawn less complicated. Furthermore, all driven equations are according to the Body frame for simplification.

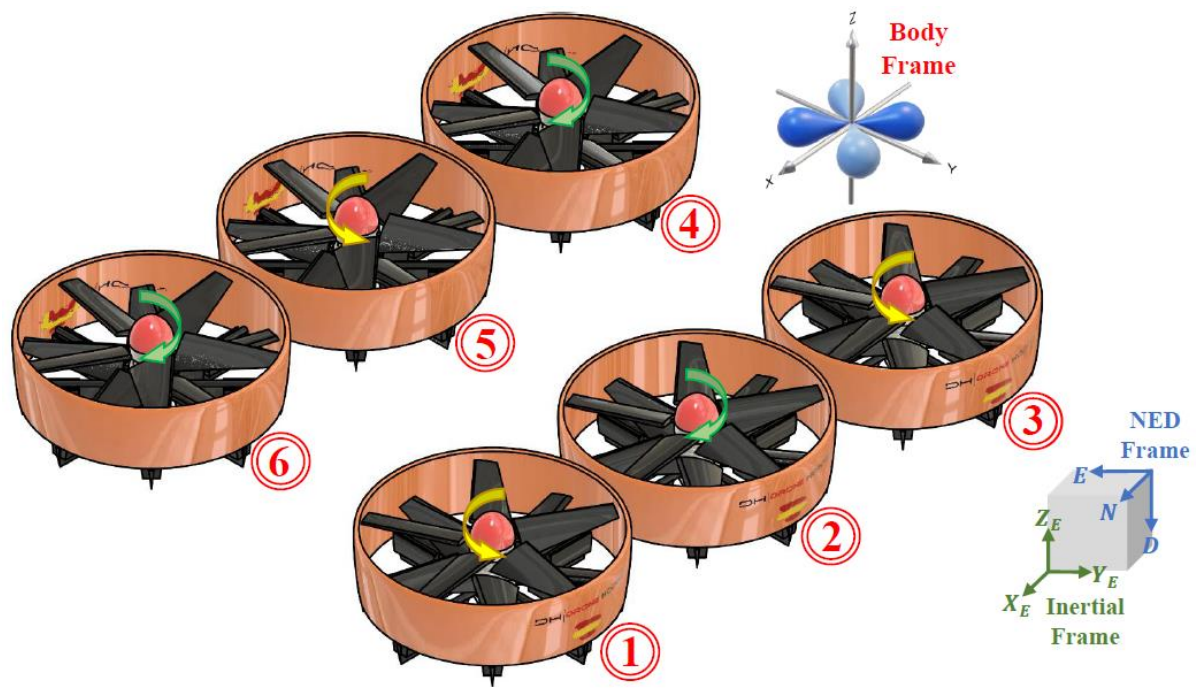

Fig. 14. Schematic of dynamical coordinate systems.

\subsection{Payload Dynamic Calculation}

WILD-HOPPER has a massive payload repository with maximally $600 \mathrm{~K}$ g of material. During rotation and, especially, abrupt maneuvers, movements of the liquid is magnificent; therefore, we supposed it as a cylindrical object with radius $r$ and $d$ long. is moving throughout the storage. The structure of the container is shown in "Fig. 15". 
Since the Center of Mass (CoM) of the drone is in upper part of the cavity, effect of $\mathrm{dh}$ moment between the CoM and center of the liquid are neglected. Far from detailed formulas of this part, we just mention the main results of Murphy and Copper's works and more detail is available through [14], [16]. Supposing the liquid viscous, we consider effects of boundary-layer terms on liquid eigenvalues [15], and their contributions for moments. Therefore, several moments are generated: pressure moment which is a the major part, because of pressure on lateral and vertical walls of the repository; wall shear moment because of viscosity of the liquid; and finally, centrifugal term which are all expressed in "Eq. (1)".

$$
\mathrm{M}_{\mathrm{pl}}=M_{\text {lat }}+M_{\text {ver }}+M_{v s}+M_{c f}
$$
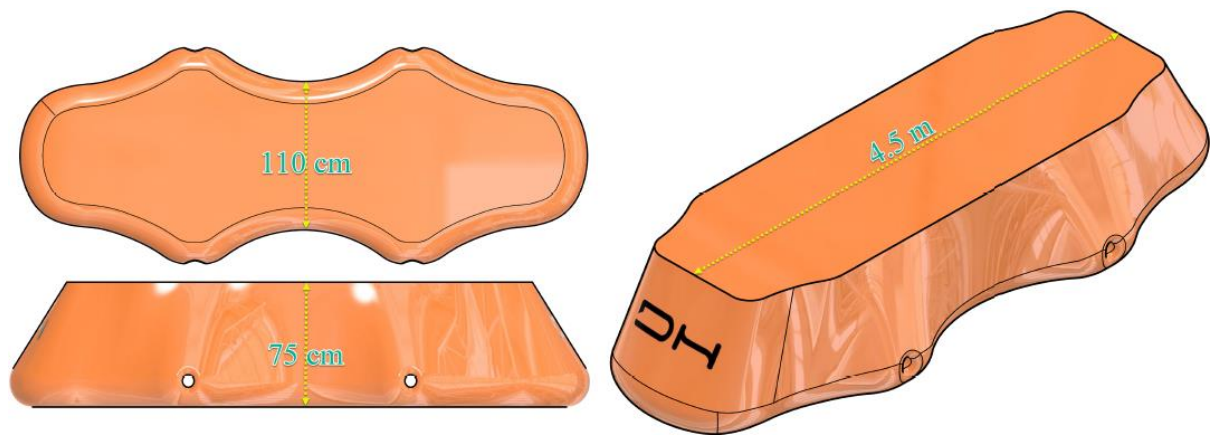

Fig. 15. Approximate structure of WILD-HOPPER liquid repository.

Wherein, $\mathrm{M} \mathrm{pl}$ is the total payload moment, $\mathrm{M}$ lat and $\mathrm{M}$ ver are the lateral and vertical parts of liquid pressure moment, Mvs viscous moment, and Mcf is centrifugal moment term, which are all brought in [14]. Clearly speaking, all the moments introduced could be distributed in two segments; slow-mode coning and fast-mode coning [17]. Slow-mode is the overall stream during spinning; nevertheless, fast-mode includes small rapid circular movements of the liquid through rolling, which both modes are shown in "Fig. 16". This solution is obtained by linearizing Navier-Stokes equations, supposing quasi-steady stream and wave motion model which produces two different spin-up model spread into slow and fast modes, which are declared through "Eq. (2)". These equations result in moments of the liquid on the fuselage.

$$
\begin{gathered}
M_{\text {total }}=M_{s m}+M_{f m} \\
\rightarrow M_{\text {total }}=m_{l} r^{2} v_{l}^{2}\left[\begin{array}{ccc}
1 & 0 & 0 \\
0 & \cos v / \omega & \sin v / \omega \\
0 & -\sin v / \omega & \cos v / \omega
\end{array}\right]\left(\begin{array}{l}
\mu_{s m} \alpha_{s m}^{2} C_{s m}+\mu_{f m} \alpha_{f m}^{2} C_{f m} \\
\mu_{s m} \alpha_{s m} C_{s m}+\mu_{f m} \alpha_{f m} C_{f m} \\
\mu_{s m} \alpha_{s m} C_{s m}+\mu_{f m} \alpha_{f m} C_{f m}
\end{array}\right)
\end{gathered}
$$

Where, $M_{\mathrm{sm}}$ and $\mathrm{M}_{\mathrm{f}} \mathrm{m}$ are slow-mode and fast-mode liquid moments, $\mathrm{m}_{1}$ is mass of the liquid, $v_{l}$ is spin rate of the liquid, $\mu_{\mathrm{sm}}$ and $\mu_{\mathrm{fm}}$ are slow and fast modes rotational frequency, $\mathrm{C}_{\mathrm{sm}}$ and $\mathrm{C}_{\mathrm{fm}}$ are the liquid slow and fast modes moment coefficient, $\alpha_{\mathrm{sm}}$, and $\alpha_{\mathrm{fm}}$ are slow and fast modes angle of attack at each cycle. 


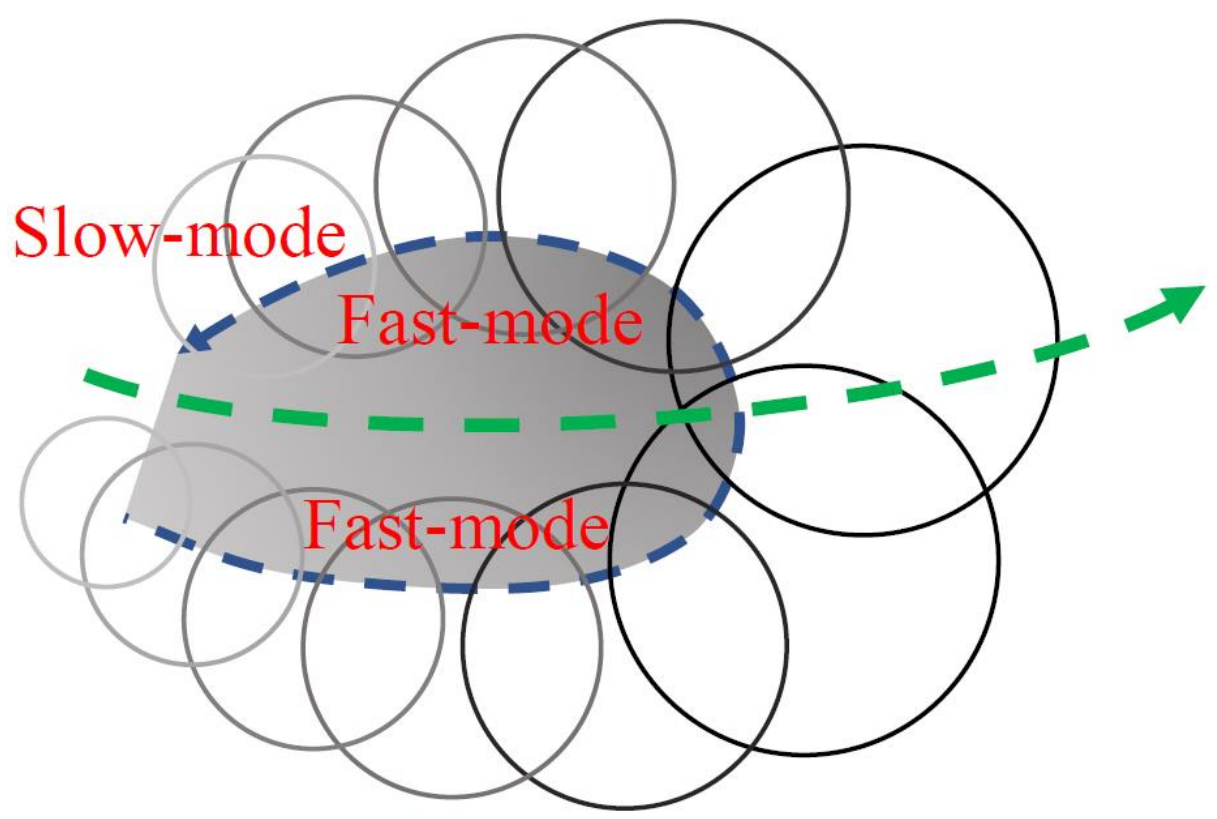

Fig. 16. Schematic of slow and fast whirling modes of the liquid inside the tank during the mission.

\section{$5 \quad$ Results}

This paper focuses on designing and implementing a new innovative Drone for forest firefighting in Europe, called WILD HOPPER, through this research we gave a deep overview for the prototype of WILD HOPPER, focusing on the limitation of the current technologies used for forest firefighting, where we developed a new patented control system and water mist jet to overcome these limitations.

Also, a deep overview for WILD HOPPER requirements where discussed in details through section 3.1 , showing the prototype design and the process used for building this prototype, by taking into consideration the operational requirements, showing the adjustment and the calibrations for the electrical and mechanical equipment used, and testing the overall system with flight test using both manual and autonomous modes for operations.

Through this research and designing process, all the regulations and system safety requirements of EASA were considered.

In addition, new software for WILD HOPPER was developed as discussed in detail through section 3.4, showing the safe parameters configuration during the mission.

Whereas, a new redundant alarm system for error monitoring was introduced with our system as shown in section 3.4.2 and "Fig. 11".

WILD HOPPER is an innovative complete system designed with fast response flight pattern execution for different missions. 
Through this research different aspects were taken into consideration starting from electrical and computer engineering implementations and tasks, until designing the dynamic model, where various results can be taken into consideration, showing the

performance of the innovated WILD-HOPPER controller. The results are brought based on two different trajectories, a quadratic $\infty$, and a sine wave path to examine the controller in sharp and smooth routes.

"Fig. 17" demonstrates the comparison of the state and reference values when the WILD-HOPPER moves from center of the coordinate system, then a square of $6 \mathrm{~m}$ length in the first part of the coordinate, and goes back to the center to do the same square in the third part (negative part). The first column plots show the position and the second ones show attitude which are all based on the time during $43 \mathrm{~s}$.

Also, the position controller response functions with least overshoot, but with delay to catch the command, which is due to the saturation implemented on attitude angles.
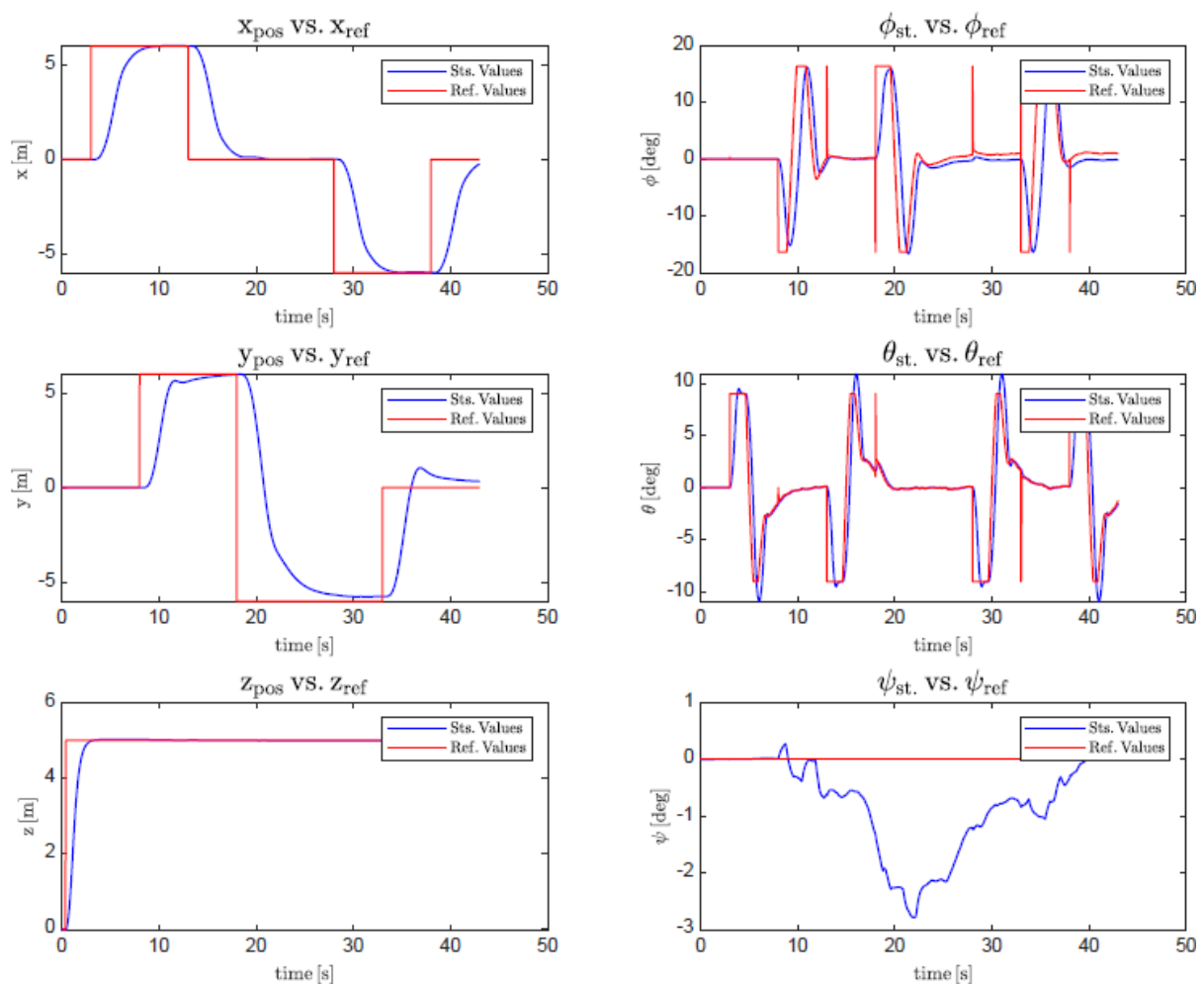

Fig. 17. Controller plot displaying the difference between state and reference values of position and attitude for WILD HOPPER.

Considering such a grand system, $\phi$ and $\Theta$ desired values are limited to $15^{\circ}$ and $10^{\circ}$ respectively, which means no maneuver more than these magnitudes is permitted. 


\section{Conclusions}

In this paper, a clear study for the forest firefighting original systems has been made, showing the deficiencies for each system. Where Drone Hopper research center highlights the needs of such a critical system by designing and implementing a new innovative drone called WILD HOPPER, solving all the shortages of the other ordinary systems.

During this research, a clear massive roadmap for the WILD HOPPER has been designed, showing the importance of the system requirements, operational requirements, and safety requirements. Through the research we have clarified the model of WILD HOPPER by changing the structure several times, obtaining the important result to include both EDF and flap systems to have a high performance in controlling attitude in such critical missions.

Furthermore, various results were brought to demonstrate the behavior of the WILDHOPPER through different trajectories as discussed in the previous section, showing the power of the WILD-HOPPER to be used in such critical missions that need high maneuvering with stability. Meanwhile, this paper will be followed by another research paper to highlight the communication and control systems of the WILD-HOPPER.

\section{$7 \quad$ Data and software availability}

All the data is available through Drone Hopper Data Center 1, Data Center 2, and the software tool used is MATLAB available at WILD-HOPPER DATA.

\section{Acknowledgment}

We would like to appreciate the European Commission for their supporting and funding of the WILD-HOPPER project, and also with great thanks to the University of Carlos III, campus Leganes, Madrid for performing laboratories and hangers through our research; and last but not least, to our colleagues at Drone Hopper for their supports during the project.

\section{References}

[1] G. Winkel, F. Aggestam, M. Sotirov, and G. Weiss "Forest Policy in the European Uion," chapter 3, European Forest Governance:Issues at Stake and the Way Forward, 2013, pp. 5263.

[2] D. Alexandrian et. al. "Forest fires in the Mediterranean area," Unasylva No. 197.

[3] J. San-Miguel-Ayanz et al. "Comprehensive Monitoring of Wildfires in Europe: The European Forest Fire Information System (EFFIS)," European Commission, Joint Research Centre Italy. https://doi.org/10.1016/j.foreco.2006.08.036 
[4] J. Vasconcelos, R. de Sousa, and P.V. Gamboa "Aerial Forest Fire Detection and Monitoring Using a Small UAV," International Congress on Engineering — Engineering for Evolution, KnE Engineering, pp. 242--256, Volume 2020. https://doi.org/10.18502/keg.v5i6.7038

[5] A. Sharma, P. Kumar Singh, A. Sharma, and R. Kumar, "An efficient architecture for the accurate detection and monitoring of an event through the sky," Computer Communications, September 2019. https://doi.org/10.1016/j.comcom.2019.09.009

[6] O.A. Saraereh, A. Alsaraira, I. Khan and R. Uthansakul, "Performance Evaluation of UAVEnabled LoRa Networks for Disaster Management Applications," Article in Sensors, April 2020. https://doi.org/10.3390/s20082396

[7] Sezgin Kilic and Omer Ozkan "A self-adaptive UAV routing for forest fire risk mitigation: a conceptual model," Modeling and Simulation International (SCS), SummerSim-SCSC, Germany, 2019.

[8] M.A. Akhlouf, A. Couturier, N.A. Castro, "Unmanned Aerial Vehicles for Wildland Fires: Sensing, Perception, Cooperation and Assistance," Drones, 2021; Vol. 5, pp. 1-25. https://doi.org/10.3390/drones5010015

[9] V. Sherstjuk, M. Zharikova, and I. Sokol, "Forest Fire-Fighting Monitoring System Based on UAV team and Remote Sensing," 38th International Conference on Electronics and Nanotechnology (IEEE ELNANO), September 2018. https://doi.org/10.1109/elnano.2018. $\underline{8477527}$

[10] E. Ausonio, P. Bagnerini, and M. Ghio "Drone Swarms in Fire Suppression Activities: A Conceptual Framework," Vol. 5, 2021. https://doi.org/10.3390/drones5010017

[11] https://www.easa.europa.eu/document-library/regulations/regulation-eu-20181139

[12] M.A. Stamate, A.F. Nicolescu, and C. Pup aža, "Study Regarding Flight Autonomy Estimation for Hexa copter Drones In Various Equipment Configurations," Proceedings in Manufacturing Systems, Vol. 15, pp. 81-90, Romania, 2020.

[13] M.S. Ale Isaac, A. Ragab, E. Caballero, P. Flore, and P. Campy, "Mathematical Modeling and Designing a Heavy Hybrid-Electric Quadcopter, Controlled by Flaps," International Conference of Autonomous Systems (IEEE ICAS), Montreal, Canada, 2021.

[14] C.H. Murphy, "Angular Motion of a Spinning Projectile with a Viscous Liquid Payload," US Army Ballistic Research Laboratory, Aberdeen Proving Ground, Maryland, August 1982.

[15] E.H. Wedemeyer, "Viscous Corrections to Stewartson's Stability Criterion," Ballistic Research Laboratory, Report 1325, 1966.

[16] G.R. Cooper, M. Costello, "The Effects of Liquid Payloads on the Trajectory of Spinning Projectiles," AIAA Atmospheric Flight Mechanics Conference and Exhibit, South Carolina, 2007. https://doi.org/10.2514/6.2007-6493

[17] J. Rogers, M. Costello, "Design Considerations for Stability of Liquid Payload Projectiles," Journal of Spacecraft and Rockets, Vol. 50, No. 1, 2013. https://doi.org/10.2514/1.a32292

[18] M.S. Ale. Isaac, S. H. Mirtajadini, and A. Naghash, "Control and Guidance of an Autonomous Quad-rotor Landing Phase on a Moving Platform," Annual Conf. Autonomous Vehicles (IMAV), Madrid, 2019.

[19] G. Cooper and M. Costello, "Trajectory Predictions of Spin Stabilized Projectiles with a Liquid Payload,” Journal of Spacecraft and Rockets, Vol. 48, No. 4, pp. 664—670, 2011. https://doi.org/10.2514/1.52564

[20] C. Xiang, W. Fan, H. Liu, B. Xu, and N. Huang, "Fan Vehicle with Tilting System," International Conference on Unmanned Aircraft Systems (ICUAS), pp. 1196-1204, Colorado, 2015. https://doi.org/10.1109/icuas.2015.7152412 
[21] V. Artale, C.L.R. Milazzo, and A. Ricciardello, "Mathematical Modeling of Hexacopter," Journal of Applied Mathematical Sciences, Vol. 7, No. 97, pp. 4805-4811, 2013. https://doi.org/10.12988/ams.2013.37385

[22] Costello, M., and Peterson, A., "Linear Theory of a Dual-Spin Projectile in Atmospheric Flight," Journal of Guidance, Control, and Dynamics, Vol. 23, No. 5, pp. 789-797, 2000. https://doi.org/10.2514/2.4639

[23] Mission Planner (Version 1.3.70) [Computer software]. Retrieved from https://ardupilot.org/planner/, 2021.

[24] QGroundControl [Computer software]. Retrieved from http://qgroundcontrol.com/, 2021.

[25] UgCS [Computer software]. Retrieved from http://ugcs.com/, 2021.

[26] Horizon [Computer software]. https://www.micropilot.com/products-horizonmp.htm, 2021.

[27] wxWidgets [C++ Libraries]. https://www.wxwidgets.org/, 2021.

[28] Q. V. Khanh, L. A. Ngoc, "An Energy-Efficient Routing Protocol for MANET in Internet of Things Environment," International Journal of Online and Biomedical Engineering (iJOE), Vol. 17, No. 07, pp. 88-99, 2021.

[29] V. K. Quy et al., "A Survey of State-of-the-Art Energy Efficiency Routing Protocols for MANET," International Journal of Interactive Mobile Technologies, 14(9): pp. 215-226, 2020.

[30] V. K. Quy, V. K. Solanki, L.A. Ngoc, “A Saving Energy MANET Routing Protocol in 5G. Secure Communication for 5G and IoT Networks," EAI/Springer Innovations in Communication and Computing series, 2021.

\section{Authors}

Ahmed Refaat Ragab is a postdoctoral engineer at the University of Carlos III, and Drone Hopper Company, Leganes, Madrid, Spain. He was graduated from Air Defense College, then got a diploma in computer engineering from the National telecommunication institute in Egypt, master degree in computer engineering from the college of engineering in the Arab Academy for Science and Technology and Maritime Transport, with an excellent degree, and the thesis title was " Secure Routing In UAV". He obtained the Degree of Doctor of Philosophy in Electrical Engineering, from the faculty of engineering, Benha University, with an excellent degree, and the thesis title was" UAV Cloud Computing System".

Mohammad Sadeq Ale Isaac is a Ph.D. student at the Polytechnic University of Madrid I, and a control engineer at Drone Hopper Company, Leganes, Madrid, Spain. He was graduated from the Khaje Nasir University of Technology, then obtained his master's in aerospace engineering, specializing in flight dynamics and control from the Polytechnic University of Tehran (Amir Kabir), and the thesis title was "Simulation and Implementation a Controlled Landing of a Quad-copter on a Moving Platform.

Marco A. Luna is a Ph.D. student at the Polytechnic University of Madrid, and a software engineer at Drone Hopper Company, Leganes, Madrid, Spain. He obtained his $\mathrm{BSc}$ in automation and control engineering from Universidad de las Fuerzas ArmadasESPE (Ecuador) and his master's in automatic and robotics from the Polytechnic University of Madrid (Spain), and the thesis title was "3D location system for aircraft inspection by UAVs". 
Pablo Flores Peña Ph.D. in Aeronautical engineer, he is the CEO \& CTO for Drone Hopper Company, he creates the research center at Drone Hopper Company. Also, he is an Aeronautical engineer \& IE MBA. He got more than 15 years of experience as chief engineer at Airbus Company.

Article submitted 2021-07-02. Resubmitted 2021-08-07. Final acceptance 2021-08-07. Final version published as submitted by the authors. 